\title{
Coupling of Feflo with Simpact
}

J. Arteaga-Gómez

R. Löhner

J. Rojek

E. Oñate 


\title{
Coupling of Feflo with Simpact
}

\author{
J. Arteaga-Gómez \\ R. Löhner \\ J. Rojek \\ E. Oñate
}

Publication CIMNE Nº-280, January 2006 
44th Aerospace Sciences Meeting and Exhibit, January 2006, Reno, NV

\title{
COUPLING OF FEFLO WITH SIMPACT
}

\author{
Joaquin Arteaga-Gomez*, Rainald Löhner ${ }^{\dagger}$, Jerzy Rojek ${ }^{\ddagger}$, and Eugenio Oñate ${ }^{\S}$ \\ School of Computational Sciences, George Mason University, $M S{ }_{4} \mathrm{Cr}$, \\ Fairfax, VA 22030-4444, USA \\ Institute of Fundamental Technological Research, \\ Warsaw, Poland \\ International Center for Numerical Methods in Engineering, \\ Universidad Politecnica de Catalunya, Barcelona, Spain
}

\begin{abstract}
This paper describes the coupling of FEFLO, a general purpose compressible and incompressible flow solver based on adaptive unstructured grids with SIMPACT, a general purpose, large deformation, explicit structural dynamics code developed at the Center for Numerical Methods in Engineering (CIMNE). Details on the codes, as well as the coupling strategy employed are given. Examples illustrate the possibilies the present fluid-structure capability offers.
\end{abstract}

\section{INTRODUCTION}

The ability to predict accurately fluid-structure interaction problems is of fundamental importance for design, analysis and reconstruction in many areas of mechanical and civil engineering. In order to characterize the effects of an accidental or intentional explosion close to a building, a coupled fluid-structure interaction calculation has to be carried out. The initial timescales of such events dictate the use of fluid and structure codes based on explicit timescales, and able to deal with strong shocks and large deformations and cracking. The present paper describes the coupling of two codes that are suitable for this class of problems: FEFLO, a general purpose CFD solver with SIMPACT, a general purpose explicit CSD code.

\section{FEFLO}

FEFLO was conceived as a general-purpose CFD code based on the following general principles:

- Use of unstructured grids (automatic grid generation and mesh refinement);

- Finite element discretization of space;

- Separate flow modules for compressible and incompressible flows;

\footnotetext{
${ }^{*}$ Research Associate, jarteaga@gmu.edu

†Professor, rlohner@gmu.edu

$\ddagger_{\text {Research Scientist, jrojek@ippt.gov.pl }}$

$\S$ Professor, onate@cimne.upc.es

Copyright (C) 2006 by the Authors. Published by the American Institute of Aeronautics and Astronautics, Inc. with permission.
} 
- ALE formulation for moving grids;

- Embedded formulation for dirty CAD/cracks/shock-structure interaction;

- Edge-based data structures for speed;

- Optimal data structures for different architectures;

- Bottom-up coding from the subroutine level to assure an open-ended, expandable architecture.

The code has had a long history of relevant blast applications ${ }^{24,1,25,2,3,4,5,6,28,7,30}$. For the prediction of blast loads, the FEM-FCT solvers ${ }^{24}$ are used, as they offer the best compromise of accuracy and speed for this class of problems.

\section{SIMPACT}

SIMPACT is a structural dynamics code that was developed at the International Center for Numerical Methods in Engineering (CIMNE), at the Polytechnic University of Catalonia (UPC). In order to respond to the increasing demand from the car industry for codes able to analyze what happens for impact/crash situations, SIMPACT was created as a general CSD code with explicit integration in time, as well as the following set of design principles:

- Lagrangian formulation;

- General large deformation analysis;

- Large, expandeable finite element library;

- Fast contact search;

- Link of FEM, SPH and DPM.

Due to its capability to simulate the contact between surfaces, SIMPACT has also been increasingly used to simulate stamping processes. SIMPACT has a complete library of CSD elements that covers the wide range of approximations/ simplicifcations required in CSD analysis. For the present work, the following element types were used:

- TRUSS: 2 node truss element.

- BEAM: 2-3 node isoparametric beam element based on the geometrically exact beam formulation in stress resultants. The total Lagrangian description is used for the element.

- QUAD4: linear 3 (triangular) or 4(quadrilateral) node solid element for plane strain/stress or axisymetric problems. The 4-noded quadrilateral element uses $2 \times 2$ Gauss point integration scheme with constant pressure in order to avoid volumetric locking, while the 3-noded linear triangular element uses 1 Gauss point and special time integration technique (split algorithm ${ }^{42}$ ) to avoid volumetric locking $^{18,19}$

- SHELQ: 4 node isoparametric quadrilateral shell element, with 2x2 Gauss integration scheme. Both the shell mid-surface and director field are interpolated with bilinear functions ${ }^{13,14}$.

- SHELT: 6 node isoparametric triangular shell element, with 3 point integration scheme. Both the shell mid-surface and director field are interpolated with quadratic functions ${ }^{13,14,37}$.

- BST: 3 node triangular shell element (Basic Shell Triangle) without rotational degrees of freedom, with one point per Gauss integration. The updated Lagrangian description is used in this element ${ }^{38}$. There is a set of elements based on the BST element included within the code ${ }^{13,14,37,38}$.

2 of 13

American Institute of Aeronautics and Astronautics Paper 2006-0696 
- SOLID: available both in linear 8 node hexahedral and 4 node tetrahedral 3D solid elements formulation for problems with large displacements and large plastic deformations. The 8-noded element uses a $2 \times 2 \times 2$ Gauss point integration scheme with constant pressure in order to avoid volumetric locking, while the 4 -noded uses a 1 Gauss point special integration technique (split algorithm ${ }^{42}$ ) to avoid the volumetric locking.

SIMPACT also incorporates a wide range of different material models, among them elastoplastic and hyperelastic. The definition of the stress-strain curve can be linear or nonlinear, and the plasticity models include different kinds of hardening. These material models will simulate properly the response of metallic materials (steel, iron, aluminum. etc). For other, more complex materials like concrete or reinforced concrete, new models have been added to the code. Among these, we mention the continuum damage model $^{34,35,36}$. Furthermore, a crack generation and progression model has been implemented in SIMPACT as part of the present work. This model is able to be used across a wide variety of materials, including steel and concrete. This crack model was identified as a prime requirement for the shock/structure interaction studies considered.

\section{COUPLING STRATEGY}

The question of how to couple CSD and CFD codes has been treated extensively in the literature ${ }^{40,23,26,9,10,27,5,21,41,22}$. Two main approaches have been pursued to date: strong coupling and loose coupling. The strong (or tight) coupling technique solves the discrete system of coupled, nonlinear equations resulting from the CFD, CSD, CTD and interface conditions in a single step. For an extreme example of the tight coupling approach, where even the discretization on the surfaces was forced to be the same, see Thornton ${ }^{40}$ and Huebner et $\mathrm{al}^{21,41,22}$. The loose coupling technique solves the same system using an iterative strategy of repeated 'CFD solution followed by CTD solution followed by CSD solution' until convergence is achieved (see Figure 1).

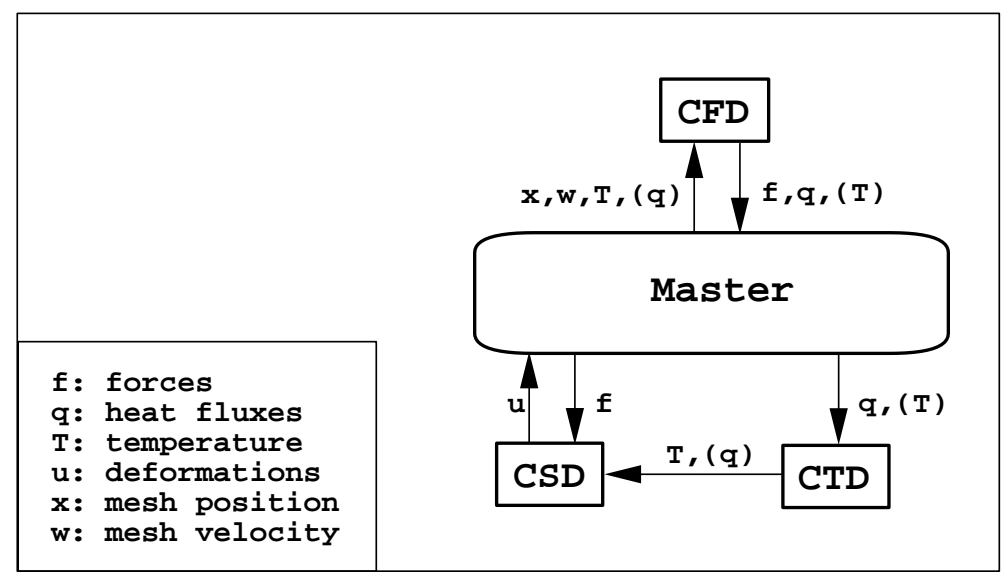

Figure 1: Loose Coupling for Fluid/Structure/Thermal Simulations

Special cases of this second approach include the direct coupling in time of explicit CFD and CSD codes and the incremental load approach of steady aero- and hydro-elasticity. The variables on the boundaries are transferred back and forth between the different codes by a master code that directs the multi-disciplinary run. Each code (CFD, CSD, CTD, ..) is seen as a subroutine, or object, that is called by the master code, or as a series of processes that communicate via message passing. This implies that the transfer of geometrical and physical information is performed between the different codes without affecting their efficiency, layout, basic functionality, and coding styles. At the same time, different CSD, CTD or CFD codes may be replaced, 
making this a very modular approach. This allows for a straightforward re-use of existing codes and the choice of the 'best model' for a given application. The information transfer software may be developed, to a large extent, independently from the CSD, CTD and CFD codes involved, again leading to modularity and software reuse. For this reason, this approach is favoured for industrialization, and used here. Indeed, cosiderable effort has been devoted to develop general, scalable information transfer libraries ${ }^{26,32,8,12,20}$. The CSD/CTD codes linked to FEFLO to date include: DYNA3D ${ }^{26}, \mathrm{PAM}^{-\mathrm{CRASH}^{33}}$, GA-DYNA ${ }^{4}, \mathrm{MARS}^{29}$, NASTRAN $^{10}$, FEHEAT $^{11}$ and FEEIGEN ${ }^{31}$.

\section{EXAMPLES}
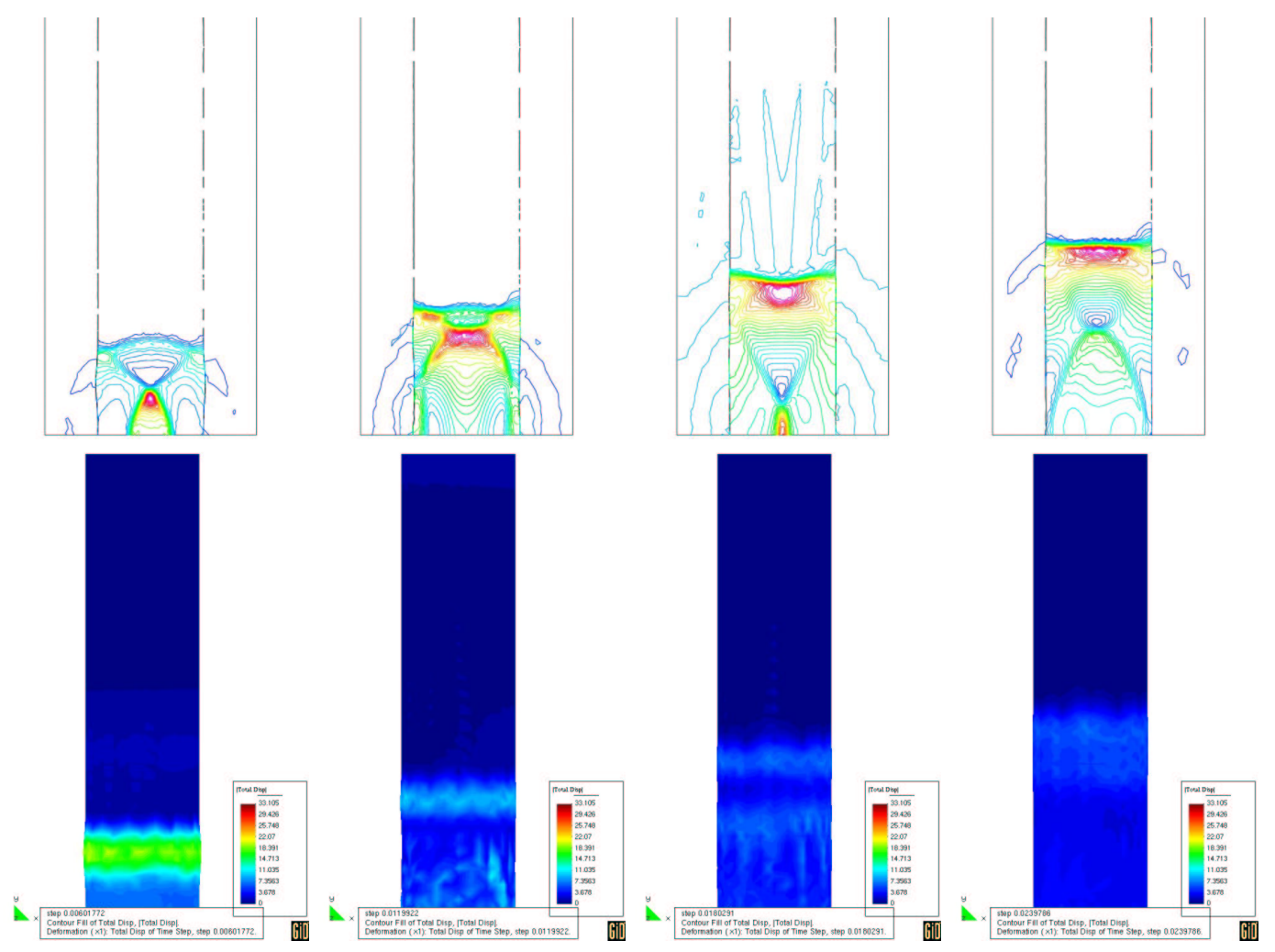

Figure 2: Blast In Tube: Elastic Case

6.1 Blast In A Tube, Elasto/Plastic: As a first example, a small blast in a tube is considered. The TNT equivalent is $100 \mathrm{~kg}$, and the tube, made of copper, has a diameter of $10 \mathrm{~m}$, a length of $40 \mathrm{~m}$, and a width of $2.48 \mathrm{~mm}$. Two CSD discretizations were considered: the first had 5476 and the second 23914 triangular (BST) shells. The material model used was elastic and elasto-plastic with the following parameters: Young's modulus: $E=117 G P a$, Poisson coefficient: $\nu=0.35$, density $\rho=8800 \mathrm{~kg} / \mathrm{m}^{3}$, yield stress: $50 \mathrm{MPa}$, fluency module: $0.1 \mathrm{Gpa}$. The CFD mesh has approximately 464000 elements, and the embedded approach ${ }^{30}$ was used. The initial solution was interpolated from a highly detailed 1-D (spherical) solution. The origin of the explosion is at the base of the cylinder. In order to provide some measure of verification, SIMPACT was replaced by SAICCSD, a similar in-house CSD code at SAIC ${ }^{39}$, while keeping the CSD discretizations the same. Figures 2,3 shows the evolution of the flowfield in the cut plane of the blast origin, as well as the surface of the CSD domain for the elastic and elasto-plastic cases for times $t=6,12,18,24 \mathrm{msec}$. Note the 
deformation due to the blast, as well as the difference in the flow solution (particularly at $t=24 \mathrm{msec}$ ) due to the fluid-structure coupling.

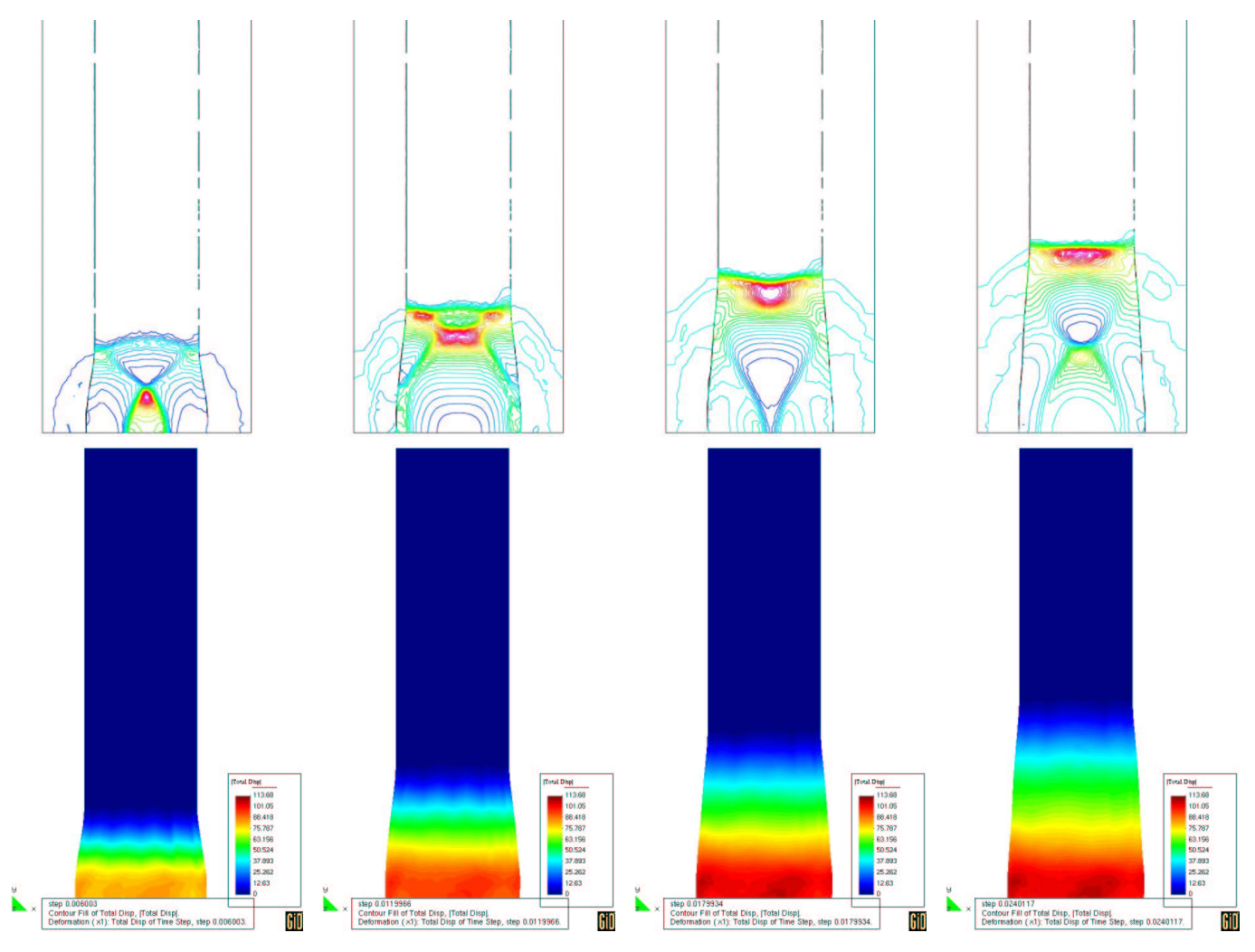

Figure 3: Blast In Tube: Elasto-Plastic Case

Figures 4a,b show a comparison of the average radial displacement at heights $h_{0}=0.0 \mathrm{~m}$ and $h_{1}=1.0 \mathrm{~m}$. Note the delay in the arrival time of the blastwave for the second of these, and the relative deformations observed. At present, an extensive series of evaluation tests are being carried out to compare the results of SIMPACT to those of DYNA3D and SAICSD, two standard codes used for this type of analysis.

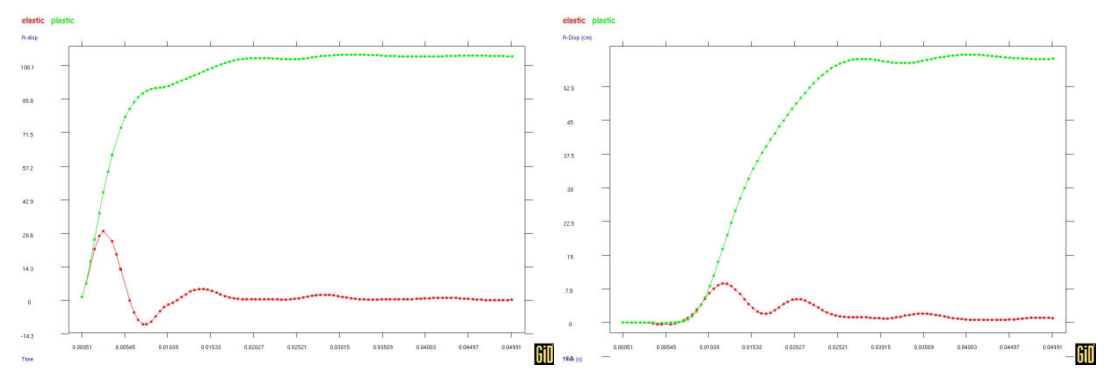

Figure 4a,b: Average Radial Displacements $(h=0,1 \mathrm{~m})$

Figures 5a-h show the average displacements and velocities for 12 points around the circumference at the base (h0) and half a meter from the base (h1). Note that as the mesh is refined, the results from SIMPACT 
and SAICCSD become closer.

Comp 2.8k disp ho

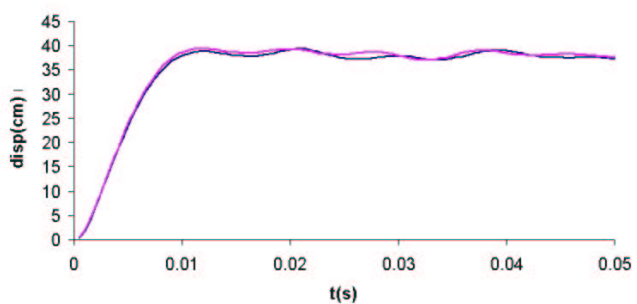

Comp 2.8k vel ho

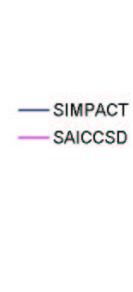

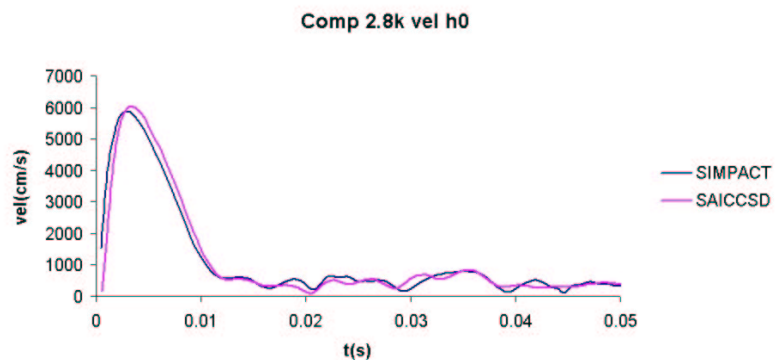

$t(s)$

Figure 5a,b: Comparison of Displacements and Velocities (nshel=5476, $h=0 \mathrm{~m}$ )
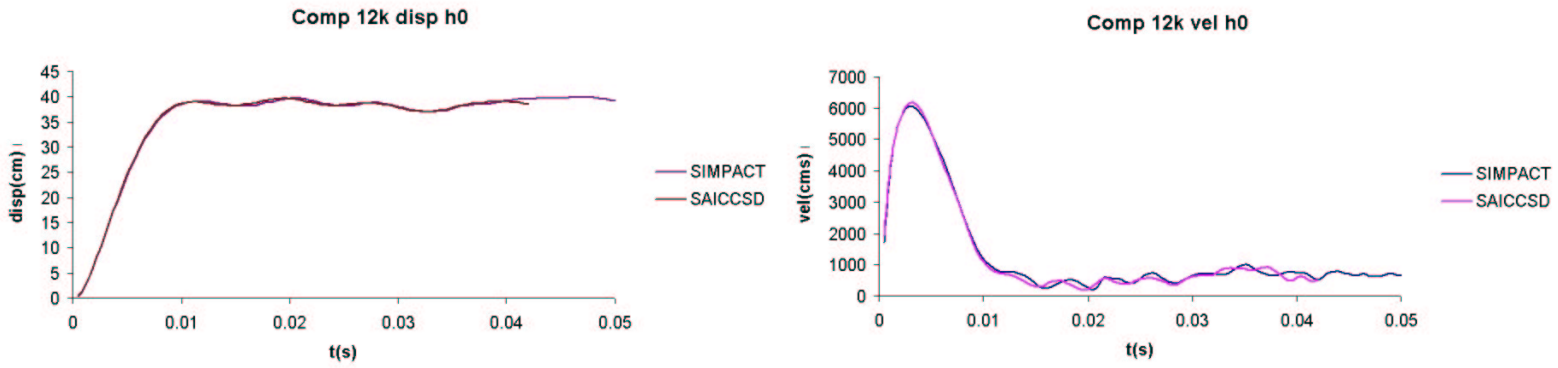

Figure 5c,d: Comparison of Displacements and Velocities $(\mathrm{nshel}=23914, h=0 \mathrm{~m})$

Comp 2.8k disp h1

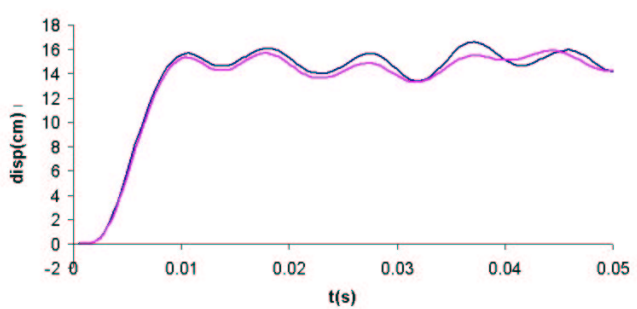

Comp 2.8k vel h1

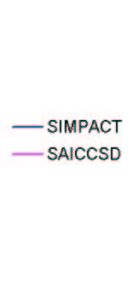

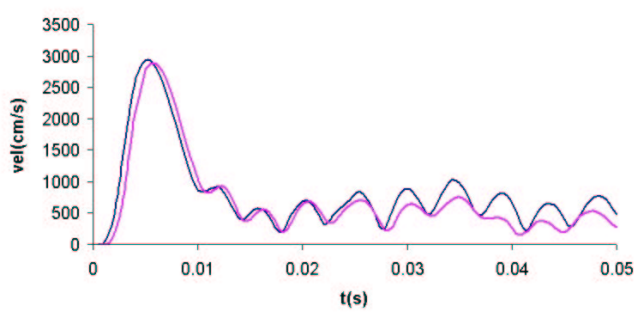

Figure 5e,f: Comparison of Displacements and Velocities (nshel $=5476, h=0.5 \mathrm{~m}$ )

Comp 12k disp h1

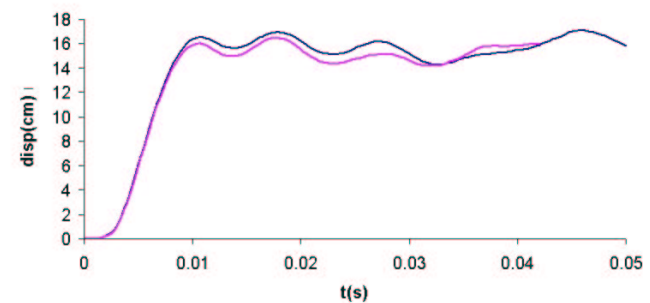

Comp 2.8k vel h1

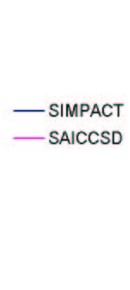

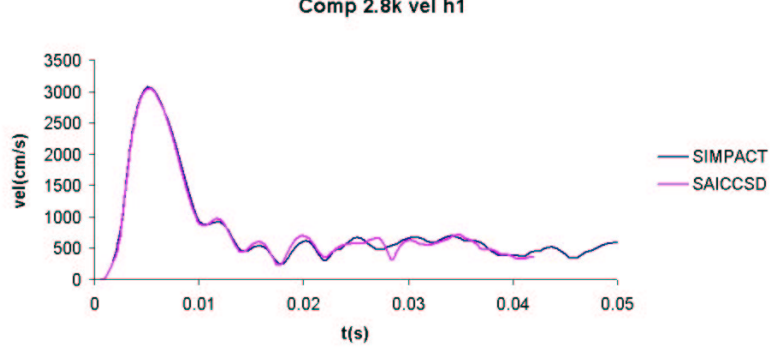

Figure 5g,h: Comparison of Displacements and Velocities (nshel $=23914, h=0.5 \mathrm{~m}$ )

6 of 13 
6.2 Blast In A Tube, Elasto/Plastic with Rupture: The ability to rupture materials during the simulation is an important requirement for the class of problems considered here. The same tube as before is considered, and the same explosive material and quantity. The thickness, however, has been reduced to $1 \mathrm{~mm}$. Two breakup methods were implemented (see Figure 6). Both of them start by computing the plastic deformation at the Gauss points. This value is interpolated to the points at every timestep. A point is considered broken if the plastic deformation exceeds the rupture deformation. For copper, we considered a value of $50 \%$. The first method (so-called free breakup) proceeds by introducing a new node for each of the elements surrounding a broken node (save one, which keeps the original node). The second method (so-called guided breakup) examines all edges (faces for volume elements) surrounding a broken node. The two with the highest tension are 'opened', and a new node is introduced. Both methods were compared for the example described above, using the finer CSD mesh. The origin of the explosion was placed at the bottom of the tube (as before). Figure 7 shows the deformation and cracking for the following times: $t=5 \mathrm{~ms}, 10 \mathrm{~ms}, 20 \mathrm{~ms}$, and Figure 8 the pressures in the mid-plane for $t=10 \mathrm{~ms}, 20 \mathrm{~ms}$. One can observe that both methods yield similar results.

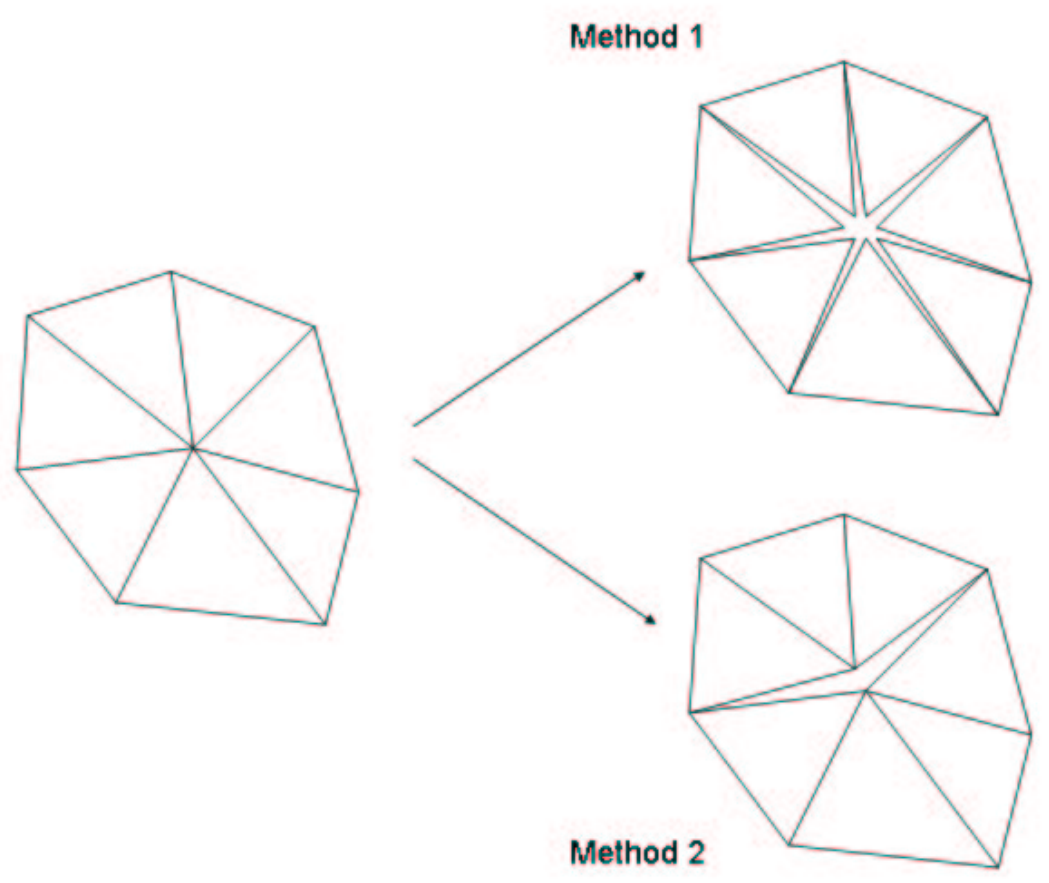

Figure 6: Breakup Methods

7 of 13 

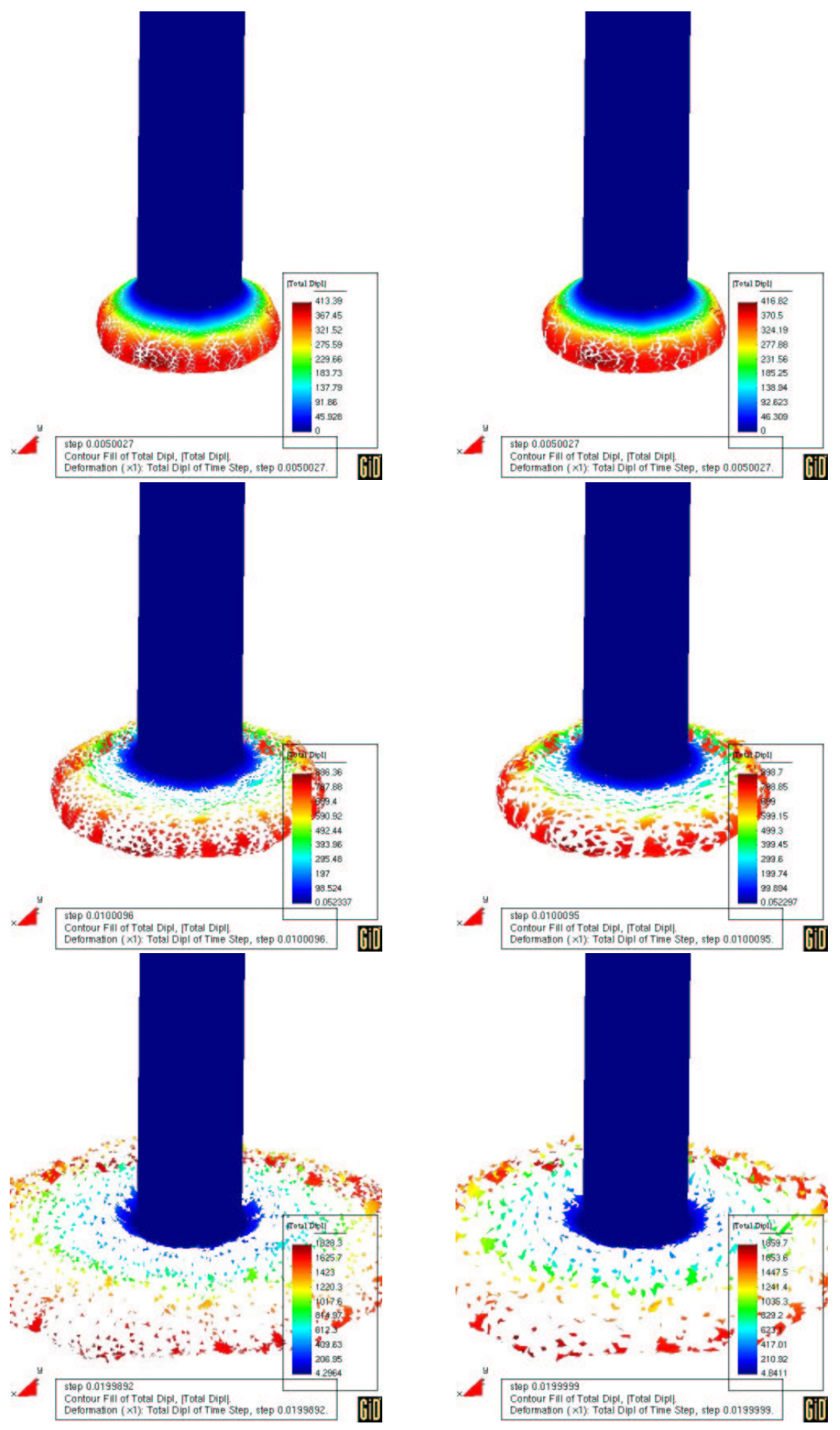

Figure 7: Breakup Patterns for Both Methods $(t=5,10,20 m s)$

8 of 13

American Institute of Aeronautics and Astronautics Paper 2006-0696 

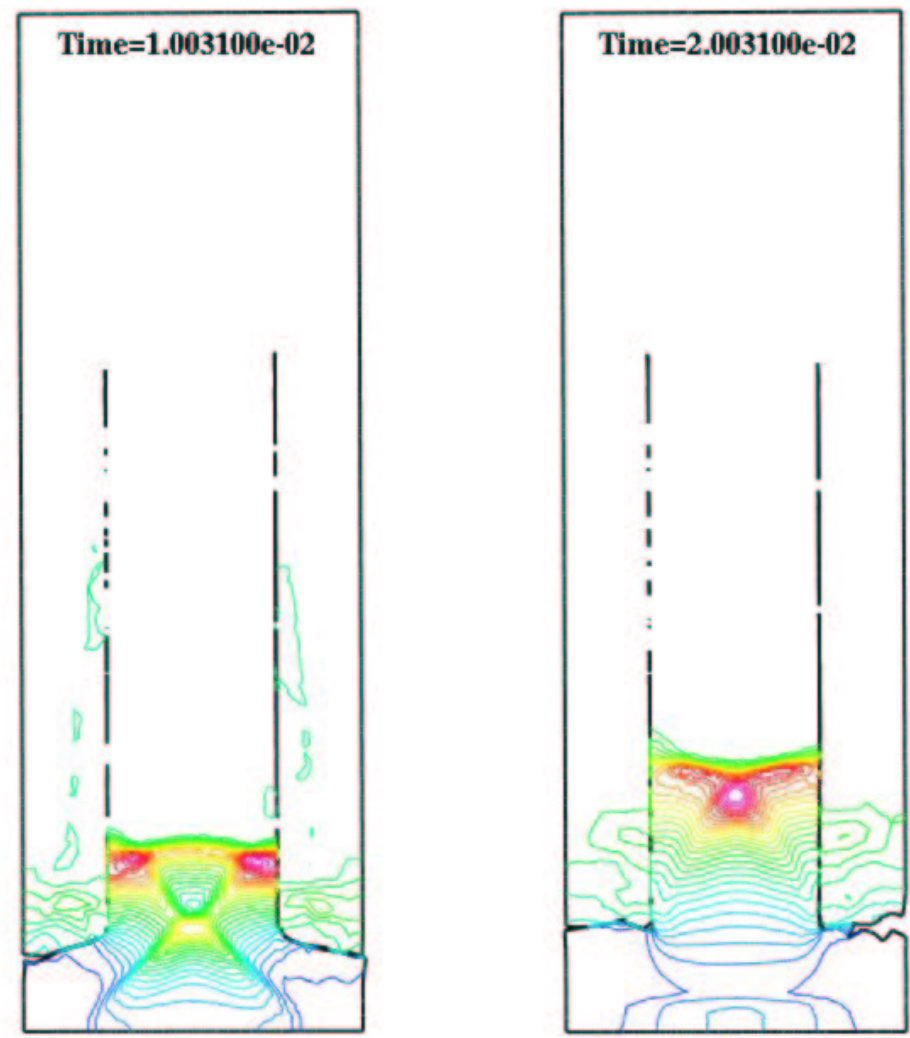

Figure 8: Pressures in Cut Plane (Method 2, $t=10,20 \mathrm{~ms})$

6.3 Blast In A Tube, Elasto/Plastic with Rupture: This is essentially the same case as before, but the explosion occurs at the center of the tube $(y=20 \mathrm{~m})$. Figure 9 shows the deformation and cracking for the following times: $t=5 \mathrm{~ms}, 10 \mathrm{~ms}, 20 \mathrm{~ms}$, and Figure 10 the pressures in the mid-plane for $t=10 \mathrm{~ms}, 20 \mathrm{~ms}$. As before, one can observe that both methods yield similar results. 

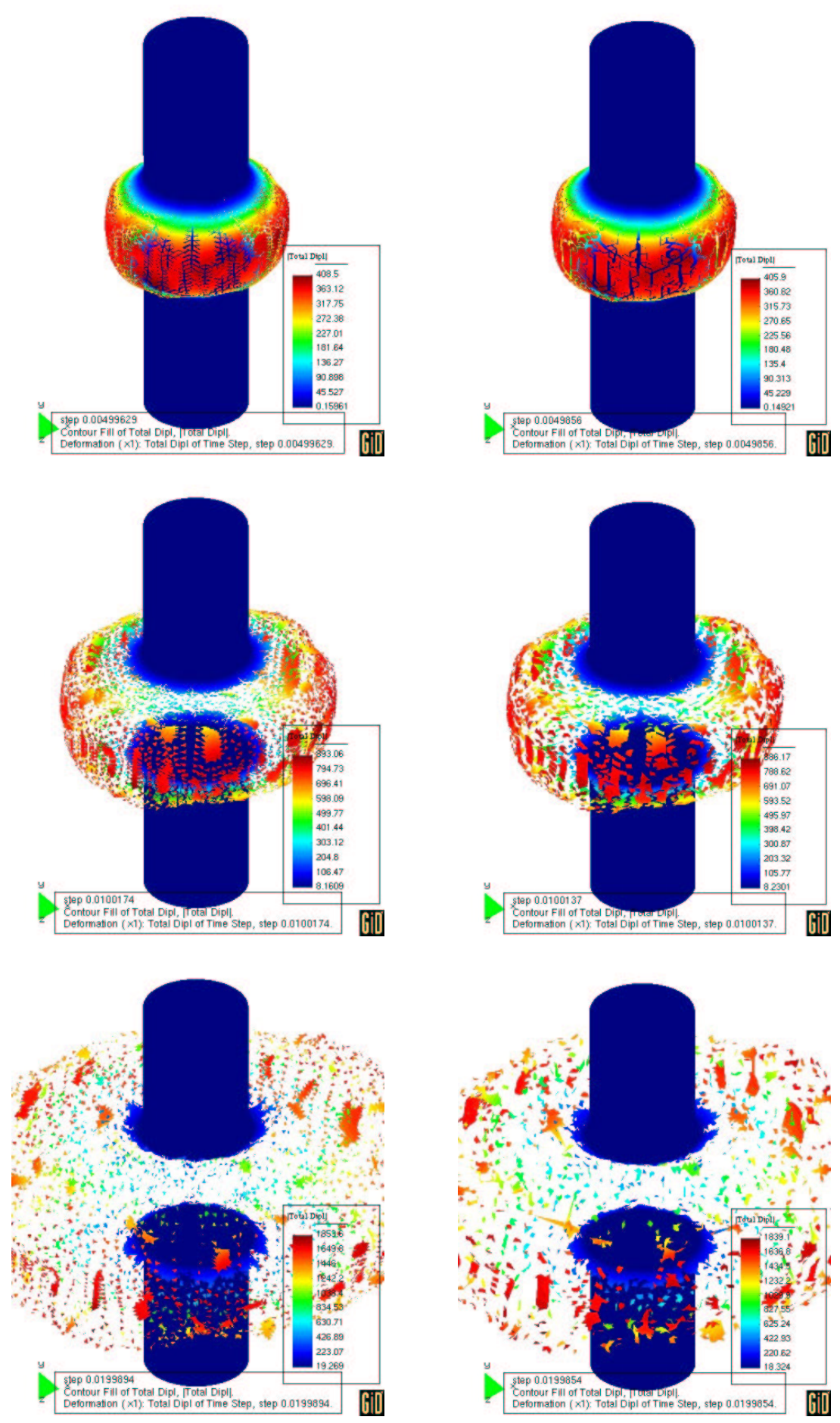

Figure 9: Breakup Patterns for Both Methods $(t=5,10,20 \mathrm{~ms})$

10 of 13

American Institute of Aeronautics and Astronautics Paper 2006-0696 

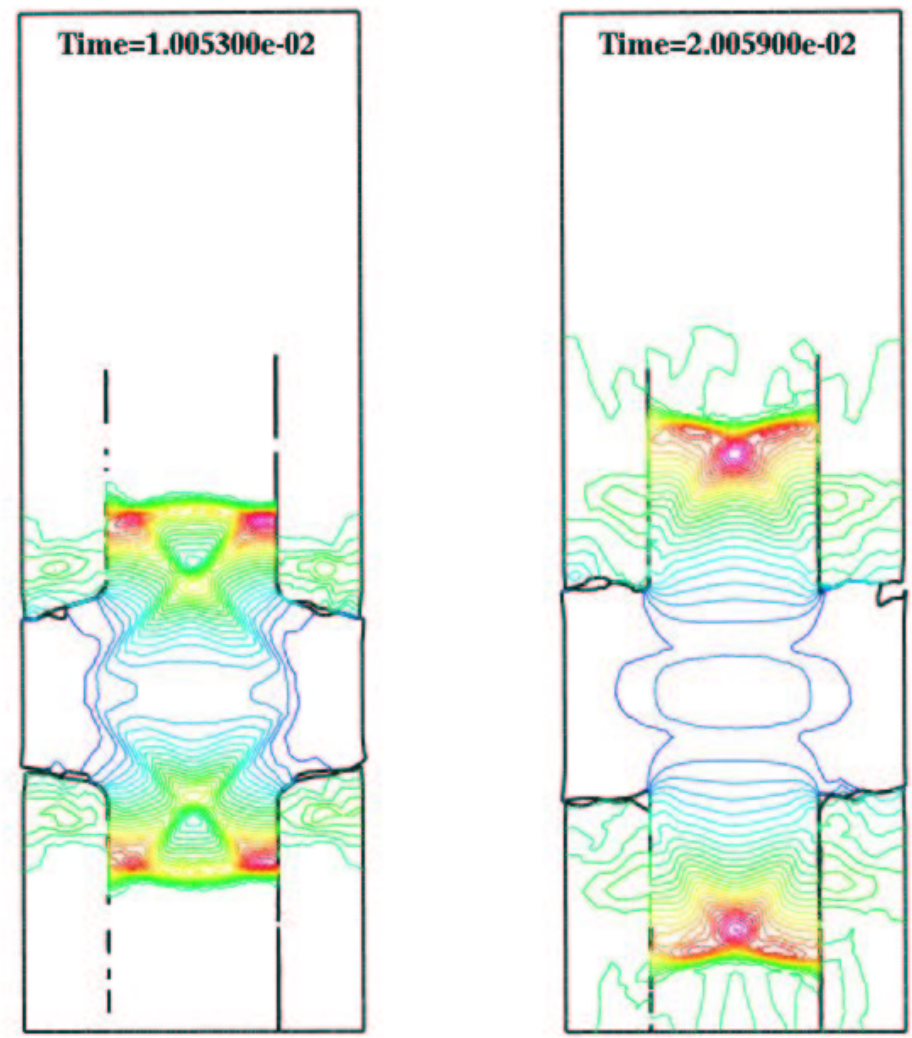

Figure 10: Pressures in Cut Plane (Method 2, $t=10,20 \mathrm{~ms}$ )

\section{CONCLUSIONS AND OUTLOOK}

FEFLO, a general purpose CFD solver based on adaptive unstructured grids has been coupled with SIMPACT, a general purpose, large deformation, explicit structural dynamics code. The coupling was done using the loose coupling approach, which was found to give satisfactory answers for the class of problems considered to date.

Like every human endeavour, numerical algorithms are subject to continuous improvements. Present research is directed at the proper treatment of:

- Cracks, particularly pressure loading in faces in gaps;

- Concrete failure; and

- Treatment of 'soft' civil engineering materials.

\section{ACKNOWLEDGEMENTS}

This research was partially supported by DTRA. Drs. Ali Amini and Seung Lee were the technical monitors.

11 of 13 


\section{References}

${ }^{1}$ J.D. Baum and R. Löhner - Numerical Simulation of Shock Interaction with a Modern Main Battlefield Tank; AIAA-911666 (1991).

${ }^{2}$ J.D. Baum. H. Luo and R. Löhner - Numerical Simulation of a Blast Inside a Boeing 747; AIAA-93-3091 (1993).

${ }^{3}$ J.D. Baum, H. Luo and R. Löhner - Numerical Simulation of Blast in the World Trade Center; AIAA-95-0085 (1995).

${ }^{4}$ J.D. Baum, H. Luo, R. Löhner, C. Yang, D. Pelessone and C. Charman - A Coupled Fluid/Structure Modeling of Shock Interaction with a Truck; AIAA-96-0795 (1996).

${ }^{5}$ J.D. Baum, H. Luo, E. Mestreau, R. Löhner, D. Pelessone and C. Charman - A Coupled CFD/CSD Methodology for Modeling Weapon Detonation and Fragmentation; AIAA-99-0794 (1999).

${ }^{6}$ J.D. Baum, H.Luo, E.L. Mestreau, D. Sharov, R. Löhner, D. Pelessone and Ch. Charman - Recent Developments of a Coupled CFD/CSD Methodology for Simulating Structural Response to Airblast and Fragment Loading; Paper presented at ICCS 2001, San Francisco, CA, May (2001).

${ }^{7}$ J.D. Baum, E. Mestreau, H. Luo, R. Löhner, D. Pelessone and Ch. Charman - Modeling Structural Response to Blast Loading Using a Coupled CFD/CSD Methodology; Proc. Des. An. Prot. Struct. Impact/ Impulsive/ Shock Loads (DAPSIL), Tokyo, Japan, December (2003).

${ }^{8}$ E. Brakkee, K. Wolf, D.P. Ho and A. Schüller - The COupled COmmunications LIBrary; pp. 155-162 in Proc. Fifth Euromicro Workshop on Parallel and Distributed Processing, London, UK, January 22-24, 1997, IEEE Computer Society Press, Lo Alamitos, Ca. (1997).

${ }^{9}$ J.R. Cebral and R. Löhner - Conservative Load Projection and Tracking for Fluid-Structure Problems; AIAA J. 35, 4, 687-692 (1997).

${ }^{10}$ J.R. Cebral and R. Löhner - Fluid-Structure Coupling: Extensions and Improvements; AIAA-97-0858 (1997).

${ }^{11}$ J.R. Cebral and R. Löhner - On the Loose Coupling of Implicit Time-Marching Codes; AIAA-05-1093 (2005).

${ }^{12}$ COCOLIB Deliverable 1.1: Specification of the COupling COmmunications LIBrary; CISPAR ESPRIT Project 20161, See http://www.pallas.de/cispar/pages/docu.htm (1997).

${ }^{13}$ F. Flores and E. Oñate - Evaluation of Different Kinds of Finite Elements Based on Simo's Shell Theory (in Spanish); Publication N. 38, CIMNE, Barcelona, Spain (1993).

${ }^{14}$ F. Flores and E. Oñate - Dynamic Analysis of Shell and Beam Structures (in Spanish); Publication N. 39, CIMNE, Barcelona, Spain (1993).

${ }^{15}$ F. Flores and E. Oñate - A Basic Thin Shell Triangle With Only Translational DOFs for Large Strain Plasticity; Int. J. Num. Meth. Eng. (2001).

${ }^{16} \mathrm{~F}$. Flores and E. Oñate - Improvements in the Membrane Behavior of the Three Node Rotation-Free BST Shell Triangle Using an Assumed Strain Approach; Comp. Meth. Appl. Mech. Eng. 194, 6-8, 907-932 (2005).

${ }^{17}$ F. Flores and E. Oñate - Advances in the Formulation of the Rotation-Free Basic Shell Triangle; Comp. Meth. Appl. Mech. Eng. 194, 21, 2406-2443 (2005).

${ }^{18}$ C. Garcia Garino and J. Oliver - A Numerical Model for Elastoplastic Large Strain Problems. Fundamentals and Applications; Computational Plasticity (D.R.J. Owen et al. eds) (1992).

${ }^{19}$ C. Garcia Garino and J. Oliver - Use of a Large Strain Elastoplastic Model for Simulation of Metal Forming Processes; NUMIFORM '92 (J.L. Chenot, R. Wood and O.C. Zienkiewicz eds), Balkeema (1992).

${ }^{20}$ GRISSLi - Numerical Simulation of Coupled Problems on Parallel Computers; BMBF-Project, Contract No. 01 IS 512 A-C/GRISSLi, Germany, See http://www.gmd.de/SCAI/scicomp/grissli/ (1998).

${ }^{21}$ B. Hübner, E. Walhorn and D. Dinkler - Numerical Investigations to Bridge Aeroelasticity; in Proc. 5th World Cong. Comp. Mech. (H.A. Mang, F.G. Rammerstorfer and J. Eberhardsteiner eds.) Vienna (2002). (see also: http://wccm.tuwien.ac.at/publications/Papers/fp81407.pdf)

${ }^{22}$ B. Hübner, E. Walhorn and D. Dinkler - A Monolithic Approach to Fluid-Structure Interaction Using Space-time Finite Elements; Comp. Meth. Appl. Mech. Eng. 193, 2087-2104 (2004).

${ }^{23}$ G.P. Guruswamy and C. Byun - Fluid-Structural Interactions Using Navier-Stokes Flow Equations Coupled with Shell Finite Element Structures; AIAA-93-3087 (1993).

${ }^{24}$ R. Löhner, K. Morgan, J. Peraire and M. Vahdati - Finite Element Flux-Corrected Transport (FEM-FCT) for the Euler and Navier-Stokes Equations; ICASE Rep. 87-4, Int. J. Num. Meth. Fluids 7, 1093-1109 (1987)

${ }^{25}$ R. Löhner and J.D. Baum - Adaptive H-Refinement on 3-D Unstructured Grids for Transient Problems; Int. J. Num. Meth. Fluids 14, 1407-1419 (1992).

${ }^{26}$ R. Löhner, C. Yang, J. Cebral, J.D. Baum, H. Luo, D. Pelessone and C. Charman - Fluid-Structure Interaction Using a Loose Coupling Algorithm and Adaptive Unstructured Grids; AIAA-95-2259 [Invited] (1995).

${ }^{27}$ R. Löhner, C. Yang, J. Cebral, J.D. Baum, H. Luo, D. Pelessone and C. Charman - Fluid-Structure-Thermal Interaction Using a Loose Coupling Algorithm and Adaptive Unstructured Grids; AIAA-98-2419 [Invited] (1998).

${ }^{28}$ R. Löhner - Applied CFD Techniques; J. Wiley \& Sons (2001).

${ }^{29}$ R. Löhner, J. Cebral, C. Yang, J.D. Baum, E. Mestreau, C. Charman and D. Pelessone - Large-Scale Fluid-Structure Interaction Simulations; Computing in Science and Engineering (CiSE) May/June'04, 27-37 (2004).

12 of 13

American Institute of Aeronautics and Astronautics Paper 2006-0696 
${ }^{30}$ R. Löhner, J.D. Baum, E. Mestreau, D. Sharov, C. Charman and D. Pelessone - Adaptive Embedded Unstructured Grid Methods; Int. J. Num. Meth. Eng. 60, 641-660 (2004).

${ }^{31}$ R. Löhner, C. Yang, and E. Oñate - On the Simulation of Flows with Violent Free Surface Motion; AIAA-06-0291 (2006).

${ }^{32}$ N. Maman and C. Farhat - Matching Fluid and Structure Meshes for Aeroelastic Computations: A Parallel Approach; Computers and Structures 54, 4, 779-785 (1995).

${ }^{33}$ E. Mestreau, V. Chen, A. Kamoulakos, and R. Löhner - Finite Element Modelling of Fluid/Structure Interaction in Explosively Loaded Aircraft Fuselage Panels Using PAMSHOCK/PAMFLOW Coupling; pp. 8.1-8.14 in Proc. Third World Conf. Appl. Computational Fluid Dynamics (A. Müller, B. Löffler, W. Habashi and M. Bercovier eds.), Basel World User Days CFD 1996, Freiburg i.Br., Germany, May (1996).

${ }^{34}$ J. Oliver - A Consistent Characteristic Length for Smeared Cracking Models; Int. J. Num. Meth. Eng. 28, 461-474 (1989).

${ }^{35}$ J. Oliver - Modeling Strong Discontinuities in Solid Mechanics via Strain Softening Constitutive Equations. Fundamentals \& Numerical Simulation; Int. J. Num. Meth. Eng. 39 (21), 3575-3623 (1996).

${ }^{36}$ J. Oliver, A.E. Huespe, M.D.G. Pulido and E. Chaves - From Continuum Mechanics to Fracture Mechanics: The Strong Discontinuity Approach; Eng. Fract. Mech. 69, 113-136 (2002).

${ }^{37}$ E. Oñate, F. Zarate and F. Flores - A Simple Triangular Element for Thick and Thin Plate and Shell Analysis; Int. J. Num. Meth. Eng. 37, 2569-2582 (1994).

${ }^{38}$ E. Oñate and F. Zarate - Rotation Free Triangular Plate and Shell Elements; Int. J. Num. Meth. Eng. 47, 557-603 (2000).

${ }^{39}$ O. Soto, J.D. Baum, R. Löhner, E.L. Mestreau and H. Luo - A CSD Finite Element Scheme for Coupled Blast Simulations; pp. 383-392 in Fluid Structure Interaction and Moving Boundaries (S.K. Chakrabati, S. Hernandez and C.A. Brebbia eds.), WIT Press (La Coruña, Spain, September) (2005).

${ }^{40}$ E.A. Thornton and P. Dechaumphai - Coupled Flow, Thermal and Structural Analysis of Aerodynamically Heated Panels; J. Aircraft 25, 11, 1052-1059 (1988).

${ }^{41}$ E. Walhorn, B. Hübner, A. Kölke and D. Dinkler - Fluid-Structure Coupling Within a Monolithic Model Involving Free Surface Flows; Proc. 2nd M.I.T. Conf. Comp. Fluid and Solid Mech. (K.J. Bathe ed.), Elsevier Science (2003).

${ }^{42}$ O.C. Zienkiewicz, J. Rojek, R.L. Taylor and M. Pastor - Triangles and Tetrahedra in Explicit Dynamic Codes for Solids; Int. J. Num. Meth. Eng. 43, 565-583 (1998).

13 of 13 